\title{
Homéostasie acidobasique : rôles du foie et du rein
}

Le rein et le foie contribuent au maintien du $\mathrm{pH}$ du milieu intérieur mais la question de la hiérarchie de leurs interventions reste posée. Les auteurs discutent ici de l'importance relative de la synthèse de l'urée, de l'ammoniogenèse et du métabolisme de la glutamine dans l'équilibre acidobasique.

\section{Patrick Vinay André Gougoux}

Professeurs à la Faculté de Médecine de Montréal.

\section{Mitchell Halperin}

Professeur à la Faculté de Médecine de Toronto.

\section{REFEERENCES}

I. Rector F. C. Acidification of the urine. In Handbook of Physiology. Section 8: Renal Physiology. Ch. 14. Washington: American Physiological Society, $1973: 431-54$.

2. Atkinson D. E., Camien M. N. The role of urea synthesis in the removal of metabolic bicarbonate and the regulation of blood $\mathrm{pH}$. Curr Top Cell Regul 1982; 2 1 : 261-303.

3. Halperin M. L., Jungas R. L. The metabolic production and renal disposal of hydrogen ions: An examination of the biochemical processes. Kidney Int. 1983, 24 : 709-13.

4. Atkinson D. E., Bourke E. The role of ureagenesis in $\mathrm{pH}$ homeostesis. Trends Biochem $\mathrm{Sci}$ 1983; $9: 296-9$.

5. Haussinger D., Gerok W., Sies H. Hepatic role in $\mathrm{pH}$ regulation: role of the intercellular glutamine cycle. Trends Biochem Sci 1984; $9: 30$-2.

6. Fine A., Carlyle J. E., Bourke E. The effects of administration of $\mathrm{HCl}, \mathrm{NH}_{4} \mathrm{Cl}$ and $\mathrm{NH}_{4} \mathrm{HCO}_{3}$ on the excretion of urea and ammonium in man. Eur 7. Clin Invest 1977; $7: 587-9$.

\section{ADRESSE}

P. Vinay : Université de Montréal. Département de physiologie. Faculté de Médecine. Case postale

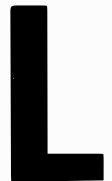

'homéostasie acidobasique du milieu intérieur est maintenue par une série de mécanismes visant à établir une relation stœchiométrique entre les ions $\mathrm{H}^{+}$produits par le métabolisme intermédiaire d'une part, et l'excrétion urinaire de $\mathrm{H}^{+}$ d'autre part [I]. Cette vision sousentend que notre régime produit des résidus acides (acide sulfurique, acide phosphorique, etc.), que la principale tâche de ce système de régulation est de nous déf endre contre une agression acide et que la sécrétion de protons par le rein régénère les bicarbonates détruits par l'arrivée des déchets acides dans le milieu intérieur [ $\mathrm{I}$ ].

De nouveaux concepts viennent cependant reposer des questions fondamentales sur les mécanismes assurant le maintien du $\mathrm{pH} d u$ milieu intérieur $[2,3]$ ainsi que sur la hiérarchie des organes (rein et foie) impliqués dans cette fonction [2].

\section{Déchets acides ou alcalins?}

Le régime d'un occidental apporte environ 3000 calories par jour. La majorité de ces calories sont fournies par les glucides et les lipides. Même si les protides ne représentent que I $5 \%$ de l'apport calorique, ce sont les protéines qui génèrent une charge acide significative [3].

Le métabolisme normal des graisses et des sucres ne produit que des déchets neutres $^{0}$ (soit de l'eau ${ }^{0}$ et du $\mathrm{CO}_{2}{ }^{0}$ )* et ne menace donc habi- tuellement pas le maintien de l'équilibre acidobasique (tabl. I). Il n'en va pas de même pour les quelque I 000 acides aminés constituant les protéines du régime qui sont à la fois porteurs d'une fonction azotée ${ }^{+}$chargée positivement et d'une fonction carboxyle ${ }^{-}$chargée négativement. Au cours de leur transformation en glucose ${ }^{0}$, en triglycéride ${ }^{0}$ ou en $\mathrm{CO}_{2}{ }^{0}$, ces acides aminés génèreront simultanément des ions ammoniaques ${ }^{+}$et des bicarbonates $^{-}$d'une façon approxi- $^{-}$ mativement stœchiométrique (tabl. II). Toutefois, certains de ces acides aminés sont porteurs d'une charge supplémentaire : leur métabolisme produira soit des protons ${ }^{+}$, soit des bicarbonates ${ }^{-}$en plus, en fonction de cette charge. Snfin, certains acides aminés soufrés tels la méthionine $^{0}$ et la cystéine ${ }^{0}$ vont produire de l'acide sulfurique au cours de leur métabolisme [3] (tabl. II).

Supposons comme Halperin et Jungas [3] que les protéines du régime correspondent au mélange d'acides aminés retrouvés dans de la viande de bœuf et examinons le résidu du métabolisme des acides aminés qu'elle contient (tabl. II). Ceux-ci produiront I I 00 millimoles de bicarbonates $^{-}$, I 000 millimoles d'ammoniaque $^{+}$et 2 ro millimoles de protons ${ }^{+}$. Il y aura en plus géné-

* La charge portée par un ion est décrit dans ce texte au moyen d'un signe $\left(^{+}\right.$, et l'absence de charge par un ${ }^{\circ}$ exemples: $\mathrm{H}^{+}, \mathrm{HCO}_{3}{ }^{-}$, glucose ${ }^{0}$. 
ration de bicarbonate résultant de l'oxydation d'environ 60 millimoles par jour de sels d'acides organiques d'origine diététique et de la libération de $\mathrm{H}^{+}$suivant l'ingestion de phosphates acides. Le tableau II montre que le résidu métabolique de l'oxydation de ces composés est largement en faveur des bicarbonates $(+930 \mathrm{mEq})$ et non des protons $(+230 \mathrm{mEq})$. Atkinson et Camien [2] ont souligné que le résidu du régime était alcalin et que le problème principal de l'homéostasie acidobasique était de défendre le milieu intérieur contre l'alcalose et non contre l'acidose.

\section{Uréogenèse et alcalose}

Les transformations métaboliques du régime ne s'arrêtent pas là : le métabolisme ultérieur de l'ammoniaque via sa transformation en urée va changer cet équilibre. Il s'agit d'un processus qui consomme de façon nette des bicarbonates [2] :

$$
\begin{aligned}
2 \mathrm{NH}_{4}{ }^{+} & +2 \mathrm{HCO}_{3}{ }^{-} \\
& \rightarrow \text { urée }^{0}+\mathrm{CO}_{2}{ }^{0}+\mathrm{H}_{2} \mathrm{O}^{0} .
\end{aligned}
$$

Ainsi, l'uréogenèse vient à la fois consommer la majorité des ions ammoniaques $^{+}$et bicarbonates ${ }^{-}$ produits par le métabolisme des acides aminés. L'équilibre net représente alors une charge acide ( $+70 \mathrm{mEq}$ ) (tabl. II). La physiologie classique a surtout mis l'accent sur les mécanismes de régénération des bicarbonates détruits par ce surplus acide, et attribue au rein un rôle central dans cette fonction [I]. Au contraire, la fonction uréogénique du foie a récemment reçu une attention particulière $[2,4,5]$ en tant que mécanisme régulateur fondamental de l'équilibre en protons par la destruction des bicarbonates produits par la diète. Ces deux visions ne s'opposent qu'en apparence, mais elles soulèvent un problème de hiérarchie : laquelle de ces deux fonctions ou lequel de ces deux organes est directement contrôlé par l'acidité des liquides corporels et joue un rôle primordial dans l'homéostasie acidobasique? Il est clair qu'un résidu diététique acide n'est retrouvé que dans la mesure où tous les ions ammoniaques $^{+}$sont métabolisés par le foie

\begin{tabular}{|c|c|}
\hline LES DÉCH & $\begin{array}{l}\text { leau I } \\
\text { DES OU ALCALINS } \\
\text { ÉGIME }\end{array}$ \\
\hline 1. glucides & déchets neutres (sauf si hypoxie) \\
\hline 2. lipides & déchets neutres (sauf si jeûne) \\
\hline 3. protides & déchets acides $>$ déchets alcalins \\
\hline 4. anions organiques & déchets alcalins \\
\hline 5. autres (phosphate, urate...) & $\begin{array}{l}\text { déchets soit acides soit alcalins } \\
\text { (mineurs) }\end{array}$ \\
\hline
\end{tabular}
en urée ${ }^{0}$. Si l'on soustrait au proces-

Tableau II

\begin{tabular}{|c|c|c|c|c|}
\hline & $\mathrm{H}^{+}$ & $\mathrm{NH}_{4}{ }^{+}$ & $\mathrm{HCO}_{3}^{-}$ & $\begin{array}{c}\text { équilibre } \\
\text { acide/base }\end{array}$ \\
\hline $\begin{array}{l}\text { A. AVANT URÉOGÉNÉSE } \\
\text { acides aminés neutres } \\
\text { acides aminés cationiques } \\
\text { acides aminés soufrés } \\
\text { acides aminés anioniques } \\
\text { anions organiques } \\
\text { phosphates acides, etc. }\end{array}$ & $\begin{array}{r}0 \\
138 \\
72 \\
\\
20\end{array}$ & $\begin{array}{r}741 \\
123 \\
36 \\
100\end{array}$ & $\begin{array}{r}741 \\
123 \\
36 \\
200 \\
\\
60\end{array}$ & : \\
\hline TOTAL & 230 & 1000 & 1160 & $=+930 \mathrm{HCO}_{3}$ \\
\hline B. APRĖS URÉOGÉNĖSE & 1230 & 0 & 1160 & $=+70 \mathrm{H}^{+}$ \\
\hline
\end{tabular}

PROTONS, BICARBONATES ET AMMONIAQUES D'ORIGINE DIÉTÉTIQUE

sus uréogénique une fraction des ammoniaques $^{+}$produits chaque jour (par exemple excrétés dans l'urine), une quantité équivalente d'ions bicarbonates générés par le métabolisme du squelette carboné des acides aminés, porteurs de ces fonctions azotées, ne sera pas titrée par le métabolisme hépatique. Cela revient à une génération nette de bicarbonates, capable de neutraliser l'excès acide et de ramener à l'équilibre le bilan de protons. La régulation de l'uréogenèse pourrait être un processus fondamental de l'homéostasie acidobasique $[2,4]$. Pour maintenir l'équilibre en protons, il apparaît plus simple de neu- traliser une partie seulement des bicarbonates produits en réglant le processus uréogénique plutôt que de faire appel à une régénération de bicarbonates faisant suite à leur destruction excessive. Cette vision s'accorde avec l'observation d'une relation inverse entre l'excrétion urinaire d'ammoniaque et d'urée [6].

Comment interpréter ce phénomène? La physiologie classique soutient que le rein répond à l'agression acide en accroissant l'excrétion urinaire d'ammoniaque $^{+}[7]$. Ceci a pour effet de soustraire du $\mathrm{NH}_{4}{ }^{+}$au processus uréogénique, diminuant ainsi indi- 


\section{REFEERENCES}

7. Pitts R. F. Production and excretion of ammonia in relation to acid-base regulation. In: Handbook of Physiology. Section 8 : Renal Physiology. Ch. I5. Washington: American Physiological Society, $1973: 455-96$.

8. Haussinger D. Hepatocyte heterogeneity in glutamine and ammonia metabolism and the role of an intercellular glutamine cycle during ureogenesis in perfused rat liver. Eur 7 Biochem 1983; 133: 269-75.

9. Vinay P., Lemieux G., Gougoux A., Halperin M. Regulation of glutamine metabolism in the dog kidney in vivo. Kidney Int. Sous presse.

10. Vinay P., Khoury N., Gougoux A. Adaptation of the rat kidney to acute acidosis: effect of tissue glutamine concentration. IXth Intermational Congress of Nephrology, Los Angeles, 1984 $177 \mathrm{~A}$.

I 1. Halperin M. L., Vinay P., Gougoux A. Pichette C., Jungas R. L. Regulation of the maxi mum rate of ammoniogenesis in the dog wit chronic metabolic acidosis: $A$ quantitative analy sis. Am 7. Physiol 1985; 248 : Sous presse.

\section{TIRES-A-PART}

P. Vinay : Université de Montréal. Département de physiologie. Faculté de Médecine. Case postale 208, Succursale A. Montréal, Qué., $\mathrm{H}_{3} \mathrm{C}_{3} \mathrm{~T} 8$. rectement la synthèse d'urée. Le rein apparaît alors comme un premier régulateur de l'homéostasie acidobasique ( $f i g$. $I$, volet gauche). Au contraire, Atkinson et Bourque [4] soutiennent que l'apport acide a diminué le capital en bicarbonate du milieu intérieur, limitant ainsi le processus uréogénique ( $f i g$. $I$, volet droit). L'excès d'azote est alors libéré dans l'urine sous forme de $\mathrm{NH}_{4}{ }^{+}$. Ici, c'est le foie qui déf end le $\mathrm{pH}$ du milieu intérieur en évitant de détruire les bicarbonates d'origine diététique. Examinons de plus près les mécanismes en cause selon ces deux hypothèses.

Rappelons que l'ammoniaque urinaire ne provient pas directement de l'ammoniaque sanguin (qui change peu avec les déséquilibres acidobasiques [7]) mais d'une production rénale locale suivant le métabolisme de la glutamine dans le tube proximal [7]. Celle-ci est synthétisée par le foie (le muscle et le cerveau) sous l'action de la glutamine synthétase [7]. Une redistribution des ammoniaques entre le cycle de l'urée et la synthèse de glutamine se produit sous l'effet d'un changement de $\mathrm{pH}$ du milieu intérieur. Le contrôle impliqué ici pourrait être lié soit à une régulation primaire de l'uréogenèse hépatique $[2,4]$, soustrayant du substrat à la glutamine synthétase ( $f i g .2$ ), soit encore à une régulation primaire de la glutamine synthétase [5] dans le foie (ou le muscle) soustrayant des ammoniaques à l'uréogenèse ( fig. I).

At tkinson et coll. ont suggéré qu'une régulation primaire de la voie uréogénique pouvait jouer un rôle déterminant dans l'équilibre en protons $[2,4]$. Selon leur modèle, les modifications du $\mathrm{pH}$ extracellulaire diminueraient (acidose) ou augmenteraient (alcalose) le rythme de synthèse de l'urée ( $f i g . I)$. Ainsi, une quantité plus ou moins grande d'ammoniaque serait détoxifiée par incorporation à la glutamine. Celle-ci serait alors exportée vers le rein où elle alimenterait le processus ammoniogénique.

Haussinger et coll. [5] proposent une autre hypothèse : le couple glutamine synthétase/glutaminase hépatique serait réglé par le $\mathrm{pH}$ systémique et contrôlerait l'uréoge- nèse. En effet, l'ammoniaque utilisé pour l'uréogenèse provient en partie de l'hydrolyse locale de la glutamine sous l'action d'une glutaminase hépatique. Une inhibition de l'activité glutaminase localisée dans les hépatocytes du territoire périportal [8] permettrait de réduire le rythme de l'uréogenèse localisée dans ces mêmes hépatocytes. Ce mécanisme, accompagné ou non d'une stimulation de l'activité glutamine synthétase localisée dans les hépatocytes centro-lobulaires [8], fournirait la glutamine nécessaire à l'ammoniogenèse rénale et réduirait la destruction des bicarbonates par le foie. Il existe des preuves expérimentales appuyant chacun des éléments de ce système.

\section{Régulation de l'ammoniogenèse}

Retenons de ces hypothèses que, quel que soit le site de régulation hépatique, un excès de glutamine doit être produit au cours de l'acidose et véhiculé au rein où il est métabolisé. Ce mécanisme implique donc que l'ammoniogenèse rénale et l'excrétion urinaire de $\mathrm{NH}_{4}{ }^{+}$suivent d'une façon ou d'une autre la synthèse de glutamine. Le foie est alors au centre du débat. Il est important de souligner ici que l'ammoniogenèse rénale n'est pas soumise à une régulation par apport de substrats. Chez l'homme, le chien et le rat, la charge en glutamine apportée au rein ne s'accroît pas au cours de l'acidose [9]. De plus, chez le rat, une surcharge en glutamine n'a qu'un effet ammoniogénique très discret, alors qu'une acidose a un effet immédiat et marqué [1o]. De nombreuses observations suggèrent d'ailleurs un effet direct de l'acidose sur le métabolisme rénal, indépendant du métabolisme hépatique. C'est le modèle classique ( $f i g .1$ ).

\section{Une expérience critique}

Afin de vérifier si le rôle principal de l'uréogenèse est de détoxifier le $\mathrm{NH}_{4}{ }^{+}$ou de détruire les bicarbonates $^{-}$, Halperin et coll. ont administré une charge en $\mathrm{NH}_{4}{ }^{+}$par voie intraveineuse à des rats normaux 
SCHÉMA CLASSIQUE (MODIFIÉ)

(capital normal en bicarbonate) ou à des rats acidotiques (capital réduit en bicarbonate); cette charge était associée soit à une charge alcaline (bicarbonate d'ammonium), soit à une charge acide (chlorure d'ammonium). Ils ont mesuré dans les quatre groupes de ce protocole le rythme et l'étendue de l'uréogenèse. Si le $\mathrm{pH}$ influence ce processus de façon significative, celle-ci devrait être plus rapide chez les animaux en alcalose et plus lente chez les animaux en acidose. Dans tous les cas, ils ont observé une synthèse rapide et équivalente d'urée, utilisant la totalité de la charge en $\mathrm{NH}_{4}{ }^{+}$administrée. Les besoins de l'équilibre en protons n'ont donc pas modifié l'uréogenèse. Cette expérience confirme bien que c'est le rein et non le foie qui détermine la distribution des azotes entre l'ammoniaque urinaire et l'urée, en accord avec les concepts classiques. Ces concepts comportent cependant des vices de compréhension : il faut réexaminer le rôle de la sécrétion de protons dans l'urine, et le rôle de l'ammoniurie dans le maintien de l'homéostasie en protons.

Classiquement [ $[, 7]$, le rein est tenu responsable du maintien de l'homéostasie en protons parce qu'il régénère, par la sécrétion de protons dans l'urine, une quantité de bicarbonates équivalente à celle qui est détruite dans le milieu intérieur par la charge acide du régime. Une translocation de $70 \mathrm{mEq}$ de protons par jour dans l'urine est finement réglée et placée sous le contrôle du $\mathrm{pH}$ du milieu intérieur. Ceci implique une excrétion de tampons puisque le $\mathrm{pH}$ urinaire minimal de 4,5 , observé chez l'homme, ne permet l'excrétion que d'une quantité minime de protons sous forme libre [I].

Le phosphate filtré échappant à la réabsorption rénale servira en partie de véhicule à ces protons : la titration de ce phosphate dans la lumière tubulaire va générer environ $30 \mathrm{mEq}$ d'acidité titrable par jour[I]. La disponibilité d'autres tampons étant très limitée, l'excrétion urinaire d'ammoniaque (environ $40 \mathrm{mEq}$ par jour) a également été mise en cause pour maintenir l'équilibre en hydrogène[I]. L'ammoniaque a ainsi été assimilée à un m/s mars 85

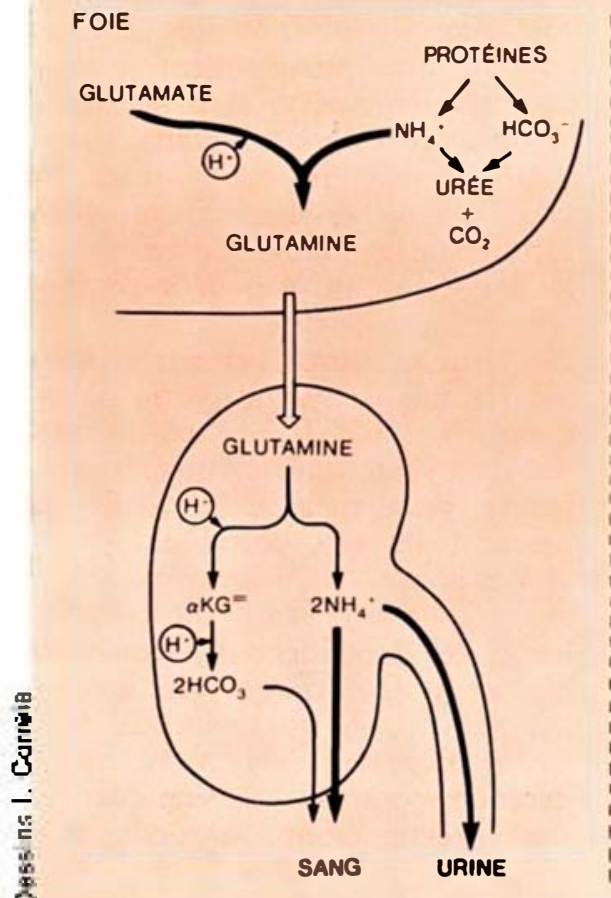

Figure 1. - La figure représente les relations entre l'ammoniogenèse et l'uréogenèse selon le schéma classique ou le schéma de Atkinson $[2,4]$ et Haussinger $[5]$. Le foie et le rein sont tous deux représentés. Les lignes en trait plein indiquent que la soustraction d'ammoniaque à l'uréogenèse contrôle cette fonction (à gauche) ou que l'équilibre en protons contrôle l'uréogenèse en dirigeant les $\mathrm{NH}_{4}{ }^{+}$non utilisés vers la synthèse de glutamine (à droite).

\section{SCHÉMA D'ATKINSON ${ }^{(2)}$ ET HAUSSINGER ${ }^{(5)}$}

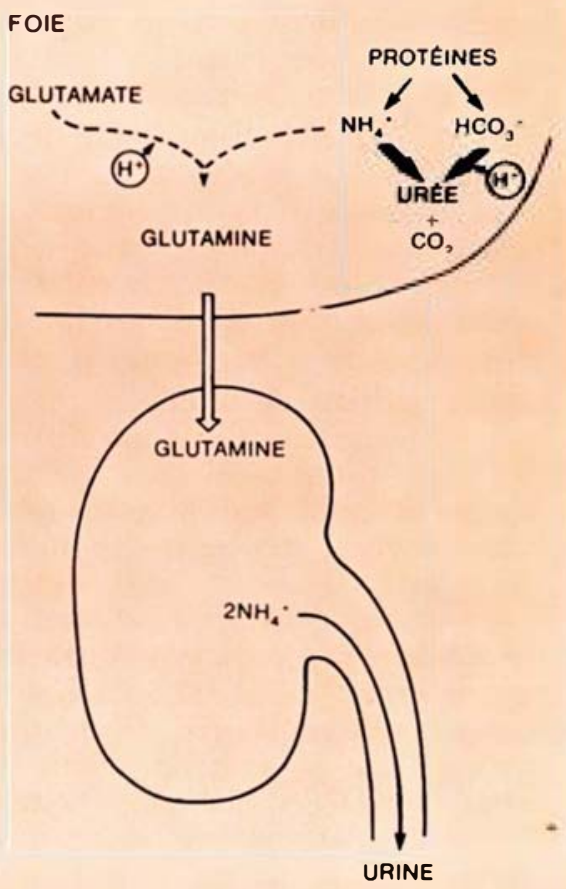

Figure 2. - La figure représente deux cellules tubulaires proximales autour de la lumière du néphron situé au centre. Les mécanismes en cause au cours de l'excrétion urinaire de $\mathrm{NH}_{4}{ }^{+}$sont décrits à gauche selon la vision classique et à droite selon la vision actuelle. Les deux schémas s'expriment de fạ̧on identique en terme d'ammoniurie. La vision actuelle propose que la translocation de protons liée à l'accumulation du $\mathrm{NH}_{4}{ }^{+}$urinaire ne joue pas de rôle direct de génération de bicarbonates dans la cellule. Ceux-ci sont produits au cours du métabolisme local de l'al phacétoglutarate.

\section{EXCRÉTION URINAIRE DE $\mathrm{NH}_{4}{ }^{+}$ \\ RÓLE POUR LA BALANCE EN H ${ }^{+}$}

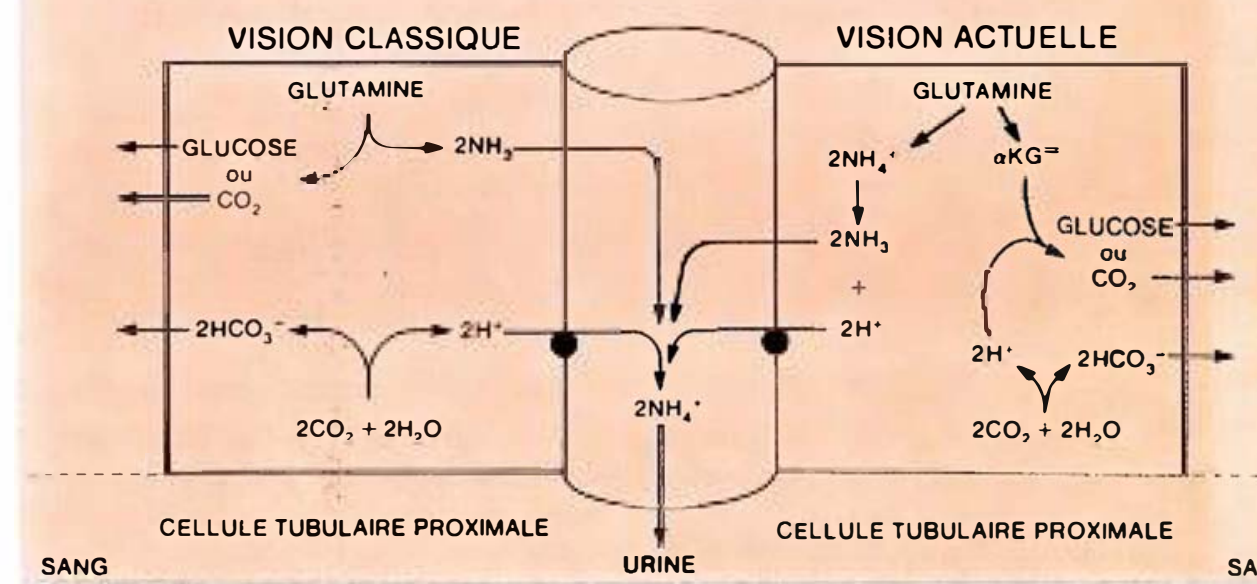


tampon urinaire [ 1,7$]$.

Selon la vision classique, l'ammoniaque est produit dans le rein sous l'effet du métabolisme local de la glutamine, un processus soumis à régulation par le $\mathrm{pH}$ systémique [7]. Ce concept est expliqué sur la partie gauche de la figure 2 : le métabo$\mathrm{NH}_{3}{ }^{0}$ dans le compartiment mitochondrial des cellules tubulaires proximales [7]. Ce gaz diffuse dans le cytoplasme des cellules impliquées, puis dans le cortex rénal tout entier [7], générant une $\mathrm{pNH}_{3}$ homogène. De là, le $\mathrm{NH}_{3}$ diffuse vers les sites d'acidification, dans le compartiment urinaire (diffusion non-ionique) où il est capté sous forme d'ammoniaque (captation ionique) par la sécrétion rénale de protons [7]. L'épithélium sécrétant à la fois un proton ${ }^{+}$et $\mathrm{NH}_{3}{ }^{0}$, il en résulte une accumulation quantitative de $\mathrm{NH}_{4}{ }^{+}$dans l'urine couplée à une génération stœchiométrique d'hydroxyles rapidement transformés en bicarbonates dans le milieu intérieur. La somme de l'ammoniaque urinaire et de l'acidité titrable représente alors l'excrétion journalière de protons par le rein, c'est-à-dire une image en miroir de la régénération journalière de bicarbonates par cet organe. Celui-ci porte alors la responsabilité de l'homéostasie acidohasique ( $f i g .2$ ).

Ce modèle est pratique et coïncide exactement avec les besoins du maintien de la balance en protons mais n'en demeure pas moins inexact à plus d'un titre. En effet, il ne tient pas compte des faits suivants : lisme de cet acide aminé produit du

l'excrétion d'acidité titrable ne correspond que partiellement à une excrétion nette de protons; le métabolisme rénal de la glutamine produit non pas du $\mathrm{NH}_{3}{ }^{0}$ mais du $\mathrm{NH}_{4}{ }^{+}$; le métabolisme du squelette carboné de la glutamine est en fait le facteur déterminant pour la génération de bicarbonates.

Halperin et Jungas[3] ont récemment souligné qu'une partie seulement (environ 50\%) des phosphates d'origine diététique est ingérée sous forme monohydrogène. Seule la titration de cette partie des phosphates pourra conduire à l'excrétion de protons provenant du métabolisme des protéines : seulement la moitié de l'excrétion urinaire de l'acidité titrable est impliquée dans ce processus (tabl. III). Cette considération contribue à souligner le rôle prépondérant de l'excrétion urinaire d'ammoniaque dans l'équilibre des protons. Or, la vision classique du rôle de celle-ci est également compromise.

\section{Rôle de l'ammoniurie}

Contrairement au mécanisme classique décrit plus haut, la production d'ammoniaque dans le milieu intracellulaire rénal se fait sous forme de $\mathrm{NH}_{4}{ }^{+}$et non de $\mathrm{NH}_{3}{ }^{0}$. L'excrétion de ce $\mathrm{NH}_{4}{ }^{+}$ne saurait donc jouer un rôle dans la régénération nette des bicarbonates. La sécrétion de protons sert alors à séquestrer ces ions dans le compartiment urinaire, un processus qui ne génère pas de nouveaux bicarbonates, comme cela

Tableau III

GÉNÉRATION DE NOUVEAUX BICARBONATES PAR LE REIN

\begin{tabular}{|c|c|c|c|}
\hline & & $\begin{array}{l}\text { génération } \\
\text { totale }\end{array}$ & $\begin{array}{l}\text { génération } \\
\text { nette }\end{array}$ \\
\hline $\begin{array}{l}\text { due à la sécrétion de } \mathrm{H}^{+} \text {libres } \\
\text { due à la formation d'acidité titrable } \\
\text { due à l'excrétion de } \mathrm{NH}_{4}{ }^{+} \text {. }\end{array}$ & & $\begin{array}{l}0.1 \\
30 \mathrm{mEq} \\
40 \mathrm{mEq}\end{array}$ & $\begin{array}{l}0.1 \\
15 \mathrm{mEq} \\
40 \mathrm{mEq}\end{array}$ \\
\hline$\because \therefore \because \because$ & TOTAL : & $70 \mathrm{mEq}$ & $55 \mathrm{mEq}$ \\
\hline
\end{tabular}

* L'excrétion de $\mathrm{NH}_{4}{ }^{+}$représente une image en miroir de la production métabolique nette de bicarbonates provenant du métabolisme du squelette carboné de la glutamine. Ces bicarbonates s'ajoutent à ceux du milieu intérieur, parce que la séquestration urinaire du $\mathrm{NH}_{4}$ prévient leur destruction par le processus uréogénique.

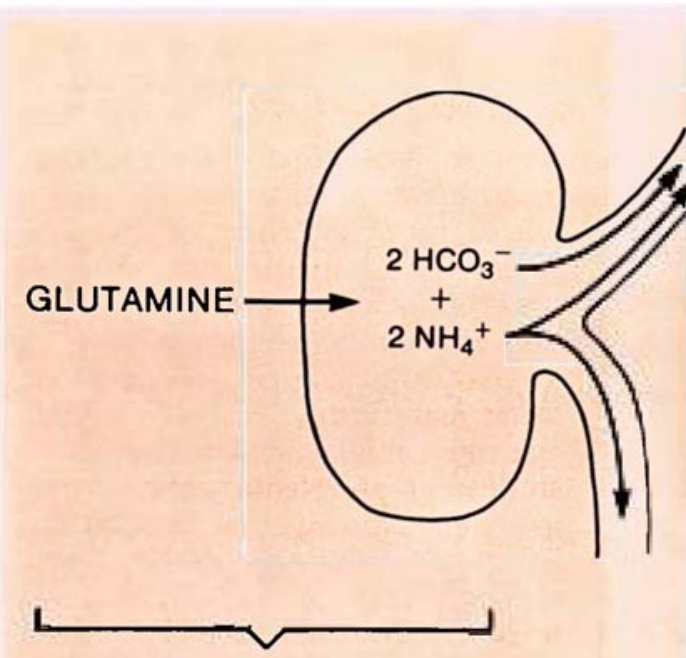

PRODUCTION DE $\mathrm{NH}_{4}^{+}$

Figure 3. - La figure montre que l'ammoniogenèse produit de façon stoechiométrique du $\mathrm{NH}_{4}{ }^{+}$et du bicarbonate ${ }^{-}$(à gauche). Ge processus aura un effet net sur l'équilibre en protons (à droite) en fonction du $\mathrm{NH}_{4}{ }^{+}$

est illustré sur la partie droite de la figure 2 . Si on ne reconsidère pas le processus de réabsorption rénale des bicarbonates filtrés, la majeure partie des protons sécrétés dans la lumière tubulaire pour défendre l'homéostasie acidobasique ne s'accompagne pas directement d'une génération de nouveaux bicarbonates dans le milieu intérieur.

On admet que c'est le métabolisme intrarénal du squelette carboné de la glutamine, l'alphacétoglutarate ${ }^{=}$, et sa transformation en corps neutres ${ }^{0}$ (c'est-à-dire en $\mathrm{CO}_{2}{ }^{\mathrm{O}}$ ou en glu$\operatorname{cose}^{0}$ ) qui va générer ces nouveaux bicarbonates. Comme cela est décrit sur la partie droite de la figure 2, ce processus consomme des protons [3]. Notons que ce schéma n'exige pas que l'alphacétoglutarate $=$ soit métabolisé dans le rein.

En accord avec les concepts énoncés plus haut et avec les mécanismes décrits sur la figure 1 , on peut donc. envisager qu'une partie des ions ammoniaques initialement portés par la glutamine et excrétés dans l'urine ne trouveront pas leur chemin vers le processus uréogénique. Les bicarbonates ${ }^{-}$produits par le 
VEINE RÉNALE:

$\mathrm{NH}_{4}^{+}+\mathrm{HCO}_{3}^{-}=$URÉE

PAS DE GAIN NET DE BICARBONATES

URINE:

GAIN NET DE BICARBONATES

L

\section{EFFET SUR LA BALANCE EN PROTONS}

séquestré dans l'urine. Le $\mathrm{NH}_{4}{ }^{+}$libéré dans la veine rénale rejoint le processus uréogénique, permettant we détruire quantitativement une partie des bicarbonates formés au cours du métabolisme de l'al phacétoglutarate ${ }^{\text {. }}$. tation urinaire s'accroît également. Ce concept est important puisqu'il attribue aux mécanismes d'acidification urinaire (surtout localisés au niveau du néphron distal) un rôle central pour la modulation fine de l'effet net du processus ammoniogénique proximal [9].

\section{Trois niveaux de régulation}

La nature a fait de la séquestration urinaire d'ammoniaque un mécanisme fondamental pour le maintien de l'homéostasie acidobasique. Afin d'assurer une souplesse maximale de fonction, trois niveaux superposés de contrôle interviennent $: 1$. La répartition du $\mathrm{NH}_{4}{ }^{+}$produit entre l'urine et la veine rénale permet de moduler immédiatement la génération nette de nouveaux bicarbonates sans faire appel à un changement du rythme du métabolisme rénal de la glutamine. Il s'agit d'une régulation portant surtout sur la sécrétion de protons dans la lumière tubulaire, en particulier au niveau distal. 2. Au cours d'une agression acide, l'extraction et l'utilisation rénales de glutamine s'accroissent, permettant une translocation maximale de $\mathrm{NH}_{4}{ }^{+}$vers l'urine. Il s'agit d'une régulation métabolique proximale réalisant une redistribution des substrats utilisés par ce segment du néphron. Chez le chien (et probablement chez l'homme) le tube proximal devient graduellement un organe exclusivement "glutaminophage" [9]. 3. Cependant, la capacité locale d'utilisation d'alphacétoglutarate produit au cours du métabolisme de la glutamine est limitée. En effet, l'utilisation métabolique de cet intermédiaire produit localement de l'ATP.

La capacité maximale d'utilisation par le rein est atteinte lorsque la totalité des besoins énergétiques (en ATP) de ce segment proximal du néphron est assurée par l'oxydation locale de la glutamine $[9,11]$. Soulignons que la fonction ammoniogénique est située dans le segment du néphron qui exerce la plus grande partie de la réabsorption sodée, principal travail du rein, où le "turnover" d'ATP est par conséquent maximal. Ceci assure la plus grande souplesse possible au métabolisme intrarénal de la glutamine

\section{Summary}

The generally accepted views on the regulation of $\mathrm{pH}$ have been recently challenged and, in particular, the respective roles of the kidney and the liver.

A new interpretation, based on the assumption that a normal diet produces more bicarbonates than protons, has shifted the emphasis on the liver and ureagenesis.

Since alkalosis and not acidosis is then the perturbing element, ureagenesis becomes the mechanism to neutralize the bicarbonates produced in excess.

Similarly, hepatic glutamine synthetase and glutaminase could modify urea synthesis according to $\mathrm{pH}$ modifications, glutamine being central in renal ammonia production.

However, recent experimental data did not confirm this hypothesis: urea synthesis was found to be similar, following a comparable nitrogen load, in acidotic or alkalotic animals, despite the opposite $\mathrm{pH}$ variations. In fact, the final product of human diet is acidic and not alkaline and the extracellular $\mathrm{pH}$ acts only indirectly on ureagenesis. but influences directly renal ammoniagenesis: ammoniuria and the distal nephron remain the primary regulators. Ureagenesis however is still essential to neutralize dietary bicarbonates. Thus both the kidney and the liver contribute to the acid base homeostasis. moniaque par le rein ne modifie l'équilibre en protons que si la cap- 